\title{
An Ontology for Secure Socio-Technical Systems ${ }^{1}$
}

\author{
Fabio Massacci, John Mylopoulos, Nicola Zannone \\ Dep. of Information and Communication Technology \\ University of Trento \\ \{massacci,jm,zannone\}@dit.unitn.it
}

\section{Introduction}

The last years have seen the emergence of standards for capturing security and privacy aspects of information systems [Ashley et al., 2003, Cranor et al., 2002, OASIS, 2005]. Those standards provide language constructs but offer no methodological tool for actually making design decisions. In this setting, the inclusion of security features within the system design is usually done after the functional design phase. This is a critical issue since security services and related protection mechanisms have to be fitted into an existing design that might be not able to accommodate them.

It is generally accepted in the Requirements Engineering (RE) research community that system development requires models that represent the system-to-be along with its intended operational environment. This is even more important when the system has to meet security requirements, since security breaches often occur at an organizational level, rather than a technical one [Anderson, 1994, Strens and Dobson, 1993]. Even though there are mature methodologies for modeling and analyzing enterprises and their organizational structure, their focus is mostly on process and marketing aspects, rather than security [AMICE Consortium, 1993, Bryce and Associates, 2006, Dignum, 2004, Fuxman et al., 2001, Hübner et al., 2002, Stader, 1996].

Socio-technical system analysis has been proposed to overcome this issue [Emery et al., 1960]. This approach aims at capturing the interactions between people and technology in workplaces. In this setting, security is the ability of the socio-technical system to protect itself against deliberate misbehavior by actors of the organizations involved in the application scenario while still providing expected services when requested by benign actors. For instance, an actor may abuse his position within the organization to gain personal advantages [House of Lords, 1999, Michaely et al., 1999, Ponemon, 2003]. Therefore, modeling and analyzing the organizational environment where the system will act is crucial for building secure systems. This allows designers to identify security mechanisms that can best protect the system, and their impacts on the system.

This chapter aims at analyzing the problem of modeling security at an organizational level. Based on such an analysis, we identify and formally define basic ontological primitives for modeling organizational and security concepts, paying particular attention to the security relevant social interaction within organizations.

\footnotetext{
${ }^{1}$ Methodological aspects of this research have been addressed in [Giorgini et al., 2005, Giorgini et al., $2006]$.
} 
To allow for a systematic design of security in organization, we have developed an agent-oriented requirements engineering methodology, Secure Tropos [Giorgini et al., 2005, Giorgini et al., 2006], tailored to describe both the organizational environment of a system and the system itself. The methodology provides a requirements analysis process that drives system designers from the acquisition of the requirements model up to its verification and validation. One of its main features is the prominent role given to early requirements analysis phase that precedes a prescriptive requirements specification. The main advantage in having such a phase is that one can capture not only the "what" or the "how", but also the "why" a software system is developed. Secure Tropos was originally based on the i* modeling framework [Yu, 1996], which is based on the concepts of actors, goals, tasks, resources, and social dependencies [Fuxman et al., 2001]. $\mathrm{i}^{*}$ has already been used to model and analyze security requirements [Liu et al., 2003]. In this work, security requirements are treated as non-functional requirements. This approach supports the representation of design decisions that can contribute to a security goal and the modeling of attackers (both internal and external) who prevent the fulfillment of goals.

However, our work revealed early on that the $i^{*}$ ontology needs to be extended in order to adequately model security, because it lacks fundamental concepts needed in order to talk about security within an organization [Giorgini et al., 2006]. To this end, we have proposed an enhanced ontology with three main notions, namely ownership, delegation and trust, which together form the very foundation of all security concerns [Giorgini et al., 2005]. Ownership is used to identify goals, tasks and resources that an actor controls; delegation is used to model the transfer of entitlements and responsibilities between actors; finally, trust represents the belief of actors about the behavior and capabilities of other actors [Mayer et al,. 1995, Rousseau et al., 1998]. Once basic ontological primitives have been identified, we develop a comprehensive ontology tailored to model security at an organizational level. To this end, we provide an axiomatic characterization of their intended semantics using Datalog [Leone et al., 2006]. The proposed ontology is intended to serve as the basis for security-related domain ontologies. From an IT perspective, it can serve as a basis for specifying together functional and security requirements.

The chapter is organized as follows. The next section reviews the current state-ofthe-art in ontologies for organization and security modeling by presenting the issues in current proposals. Section 3 introduces a bank scenario used as a running example to illustrate the application of the proposed ontology. Section 4 introduces a set of primitive concepts for modeling security at organizational level. Section 5 presents an axiomatic theory of the identified primitives. Section 6 shows how the introduced concepts are enough to detect security vulnerabilities. Finally, Section 7 concludes the paper with some directions for future work.

\section{Related Work}

Several research communities have approached the problem of enterprise modeling and analysis, and some of these have addressed issues of security. We discuss below some of the more prominent approaches. 
Enterprise Engineering. Organizational modeling of enterprises is often dealt with by enterprise engineering methodologies [AMICE Consortium, 1993, Bernus and Nemes, 1996, Bryce and Associates, 2006, Stader, 1996]. Each methodology includes an ontology for modeling organizations, usually supported by a modeling environment and various analysis tools.

Multi-Agent Systems (MAS). Efforts towards modeling organizations have also originated in the MAS community [Dignum, 2004, Hübner et al., 2002]. These approaches propose to model multi-agent systems as organizational structures.

Semantic Web. Ontologies constitute basic infrastructure for the Semantic Web. The idea underling Semantic Web proposals is to use shared vocabularies for describing entities of the domain and their inter-relationships [Masolo et al., 2004].

Security Engineering. One of main challenges of security is data protection. Resources must be protected against unauthorized access and/or tampering. This has spurred many researchers to define languages tailored to model privacy and access control policies [OASIS, 2005, Ashley et al., 2003, Cranor et al., 2002].

Enterprise engineering approaches tackle the issues of organizational analysis and modeling from an enterprise perspective. For instance, the Enterprise Project [Stader, 1996] is intended to capture an enterprise-wide perspective of organizations. Such models are intended to drive enterprises in making strategic, tactical and operational decisions. To achieve a high degree of integration, the Enterprise Project has proposed the Enterprise Ontology [Uschold et al., 1998] which includes a set of terms often used to describe enterprises. In particular, the ontology focuses on organizational structure, strategy, activities and processes, as well as marketing aspects. The Enterprise Engineering Methodology [Bryce and Associates, 2006] provides a framework that allows the study of an organization and the development of an enterprise strategy synchronized with organizational goals. The methodology includes an ontology for specifying priorities within an organization, along with plans for implementing them.

The Computer-Integrated Manufacturing Open-System Architecture (CIMOSA) [AMICE Consortium, 1993] aims at integrating enterprise operations by means of efficient information exchange within the enterprise. CIMOSA models enterprises using four perspectives: the function view describes the functional structure required to satisfy the objectives of an enterprise and related control structures; the information view describes the information required by each function; the resource view describes the resources and their relations to functional and control structures; and the organization view describes the enterprise organizational structure, i.e., the responsibilities assigned to individuals for functional and control structures. The Generalised Enterprise Reference Architecture and Methodology (GERAM) [Bernus and Nemes, 1996] defines a set of concepts for designing and maintaining enterprises during their entire life-history spanning from products to enterprise integration and strategic enterprise management. This framework identifies basic concepts used to describe the structure, content, and behavior of enterprises. Such concepts enable the modeling of the human component in an enterprise operation as well as the parts of business processes and their supporting technologies.

Among proposals from the multi-agent systems domain, OperA [Dignum, 2004] aims at designing models of organizations that support dynamic and autonomous interactions by focusing on agent societies. This proposal uses the agent paradigm to 
provide a natural way to view and characterize intelligent organizational systems. To model different roles, goals and interactions within an organization, the framework adopts a 3-layer approach: the organizational model describes the intended behavior and overall structure of the society from the perspective of the organization in terms of roles, interactions and social norms; the social model instantiates the organizational model with specific agents mapped to roles through a social contract; finally, the interaction model describes the society agents interactions by the means of interaction contracts. The OperA framework is supported by a Logic for Contract Representation (LCR), a language based on deontic temporal logic that provides a formal framework and integrated semantics at all three levels of society specification. MOISE+ [Hübner et al., 2002] focuses on the structure and functionalities of an organization, and the deontic relation between them in order to explain how a MAS achieves its purpose. Accordingly, the organizational specification is formed by a structural specification, a functional specification, and a deontic specification. The structural specification adopts the concepts of role, role relation, and groups to model the individual, social, and collective structural levels of organizations. The functional specification is based on the concepts of missions (a set of global goals) and global plans (the goals in a structure). The deontic specification then links the structural specification to functional specification in terms of permissions and obligations.

The Tropos methodology [Bresciani, 2004] is an agent-oriented software engineering methodology intended to support all analysis and design activities in the software development process. The methodology consists of five phases, namely Early Requirements, Late Requirements, Architectural Design, Detailed Design, and Implementation. Early Requirements aims at understanding the domain with its stakeholders and their individual and shared goals. Late Requirements focuses on the elicitation of requirements for the system-to-be. Architectural Design specifies the system architecture in terms of a set of interacting software agents. Detailed Design is concerned with the specification of agent capabilities and interaction. Finally, Implementation deals with the production of code from the detailed design specification. The Tropos methodology adopts the $i^{*}$ modeling language [Yu, 1996], which allows designers to model the organizational environment of a system and the system itself. This language offers primitive concepts such as actor, goal, plan, resource, as well as social dependency relationships between two actors. A goal represents strategic interests of an actor. A plan specifies a particular course of action that produces a desired effect, and can be executed in order to satisfy a goal. A resource represents a physical or an informational entity. Finally, a dependency between two actors indicates that one actor depends on another to accomplish a goal, execute a plan, or deliver a resource. The modeling framework of $i *$ includes strategic dependency models for describing the network of inter-dependencies among actors, as well as strategic rationale models for describing and supporting the reasoning of each actor vis-a-vis other actors.

Among proposals for Semantic Web, we note the Descriptive Ontology for Linguistic and Cognitive Engineering (DOLCE) [Masolo et al., 2004]. DOLCE aims to capture ontological categories that underlie natural language and human common sense. Such an ontology uses three main entities for modeling organizational settings: organizations, norms and roles. Norms describe the structure and purposes of an organization by identifying its main concerns and the behavior of its agents. The link 
between agents and norms is represented in terms of roles. In DOLCE, agents must perform specific activities because they are associated with particular roles.

In the realm of security and privacy modeling, we find sophisticated proposals such as XACML [OASIS, 2005], EPAL [Ashley et al., 2003], and P3P [Cranor et al., 2002]. XACML is an OASIS standard supporting both an access control policy language and an access control decision language. XACML defines schemes for the specification of both a context and access control policies. The context schema defines how to represent access requests in terms of the attributes describing a subject, a resource, an action, and an environment, whereas the policy schema defines how to represent access control policies in terms of access permissions and access denials. An EPAL policy is essentially a set of privacy rules that includes a data user, an action, a data category, and a purpose with conditions and obligations. On the other hand, P3P aims at formalizing privacy statements that are published by an enterprise. The goal is to define a machine-readable equivalent for the human readable privacy promises that are published as a privacy statement on a web page. Unlike XACML and EPAL, P3P defines a global terminology that can be used to describe privacy policies for an enterprise. However, these standards do not address issues of design: the system administrator must manually decide which is the right policy to protect the information system he is responsible for. Moreover, these proposals do not provide facilities for modeling the structure of an organization together with organizational goals. Accordingly, it is not possible to verify whether a given policy is consistent with the functionalities of the system.

Requirements Engineering usually treats security as a non-functional requirement [Chung et al., 2000]. Non-functional requirements introduce quality characteristics, but they also represent constraints under which the system must operate [Sommerville, 2001]. Although system designers have recognized the need to integrate most of the non-functional requirements, such as reliability and performance, into the software development process [Dardenne et al., 1993], security is still an afterthought. The usual approach is to identify security requirements after the definition of the functional design. This attitude may lead to generating serious design challenges that usually translate into software vulnerabilities or serious organizational blunders.

Security needs are generically expressed by organizational security policies. An organization defines high-level policies about security with respect to its strategic objectives and its organizational structure. Such policies have to be mapped to the specific functionalities of their information systems. Without an explicit model of the organization and the trust relationships among its components it can be result particularly complex to find the reasons that have motivated their introduction [Lampson, 2004]. For instance, ignoring trust concerns seriously affects the effectiveness of security measures imposed on a system. Such measures might be either excessive in some cases or inadequate in others. For instance, system designers may not introduce security measures since they may implicitly assume trust relationships among users that are in fact not there in the domain. Alternatively, system designers may introduce expensive mechanisms for protecting a trusted system that has not been perceived as such by designers. 
The purpose of this chapter is to define a novel ontology supporting the integration of security and requirements engineering during early phases of system development. Such an ontology is intended to aid designers in understanding why security mechanisms such as authentication, access control, or back ups are necessary, and once they are selected, what are the trade-offs from the standpoint of corporate missions. Indeed, current methodologies for software development do not meet the needs for resolving security problems [Tryfonas et al., 2001], and fail to provide evidence of integrating successfully security concerns thought the whole development process. Although there have been several proposals for modeling security features, what is still missing are models that focus on high-level security concerns, i.e., models that do not force designers to immediately get down to security mechanisms.

For instance, Jürjens has proposed UMLsec [Jürjens, 2004] for modeling security related features, such as confidentiality and access control. Basin et al. proposed an UML-based modeling language, SecureUML [Basin et al., 2006]. Their approach is focused on modeling access control policies and integrating them into a model-driven software development process. McDermott and Fox adapt use cases to capture and analyze security requirements, and they call these abuse cases [McDermott and Fox, 1999]. An abuse case is an interaction between a system and one or more actors, where the results of the interaction are harmful to the system, or one of the stakeholders of the system. Guttorm and Opdahl define misuse cases, the converse of UML use cases, which describe uses that the system should not allow [Sindre and Opdahl, 2005].

\section{A Running Example}

A major source of vulnerabilities is due to the presence of conflicts and loopholes at the interface between an IT system and its operational environment. Only by analyzing the system from an organizational perspective designers can identify appropriate security solutions.

An application domain where such issues are prominent is the banking domain. Banks, by their very nature, have to enforce security in the context of distributed control and responsibility, also evolving services and infrastructures. Protection measures, such as access control policies, separation of duties, auditing, nonrepudiation action, digital signatures, all need to be considered and applied to comply with security and legal requirements besides functional requirements for a system-tobe.

In this chapter, we focus on a banking scenario and more specifically on loan process in the context of which activities take place and assignment of rights, roles, and tasks need to be carefully considered from a security perspective. In this scenario, we are going to emphasize the necessity of preventing frauds, preserving data integrity, and protecting customer privacy rights.

\section{$4 \mathrm{Si}^{*}$ : A Language for SRE}

The definition of a modeling language for designing secure socio-technical systems includes the definition of primitive concepts for modeling organizational and security concerns, as well as the logical formalization of such primitives. Our language, $\mathrm{Si}^{*}$ 
(Secure $i^{*}$ ), is based on the $i^{*}$ ontology [Fuxman et al., 2001], where specifications employ basic primitives such as "actor”, "role”, "goal”, “task”, “resource”, and "social relationships between actors".

\subsection{Actors and their specializations}

An actor is an active entity that has strategic goals and performs actions to achieve them. Actors can be decomposed into sub-units for modeling the internal structure of organizations. Complex social actors can be modeled using two types of sub-units: agents, and roles. An agent is an actor with concrete, physical manifestations. The term agent can be used to refer to human as well as software agents and organizations. A role is the abstract characterization of the behavior of a social actor within some specialized context. Figure 1 shows the graphical representation of actors and their specializations.
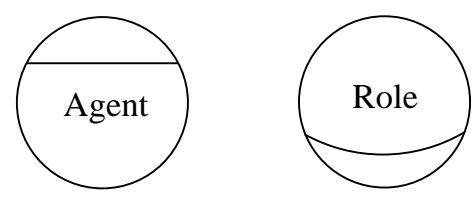

Figure $1 \mathrm{Si}^{*}$ graphical representation of agents and roles

An agent is said to play a role. The play relation is similar in the intuition to the user-role assignment of the RBAC approach [Sandhu et al., 1996]. According to such an approach, an agent inherits the properties of the roles he plays. Agents and roles can be further analyzed by decomposing them using the relation is part of. For instance, this relation can be used to identify the member of an organization as well as the sub-components of a software agent.

$\mathrm{Si}^{*}$ provides support for modeling role hierarchies based on the concept of specialization. A role is a specialization of another if it refers to more specialized activities. In this setting, all specialized sub-roles inherit all properties of the generalized super-role.

Together with the notion of specialization, $\mathrm{Si}^{*}$ adopts the notion of supervision from [Moffett, 1998]. Actually, a role hierarchy based on specialization does not correspond to the organizational structure of systems. The basic idea underlying supervision is that, if a role supervises another role, the first is responsible for the behavior of the latter and has the capabilities to control and evaluate the latter's work. This concept is used to build the organizational hierarchy (or organization chart) (Figure 3), whereas the specialization role hierarchy is built by using the ISA relation (Figure 2).

Example 1 The director of a bank is responsible for the correct delivery of the service offered by bank itself. The director cannot perform all such services by himself, and so appoints managers and clerks (e.g., pre-processing clerks and post-processing clerks) to perform some of the tasks he is responsible for. If services are not provided in compliance with bank policies, he is personally liable. Thereby, the director has good reasons to check and evaluate the behavior of subordinate roles. Figures 2 and 3 represent the roles presented above and the relations between them. 


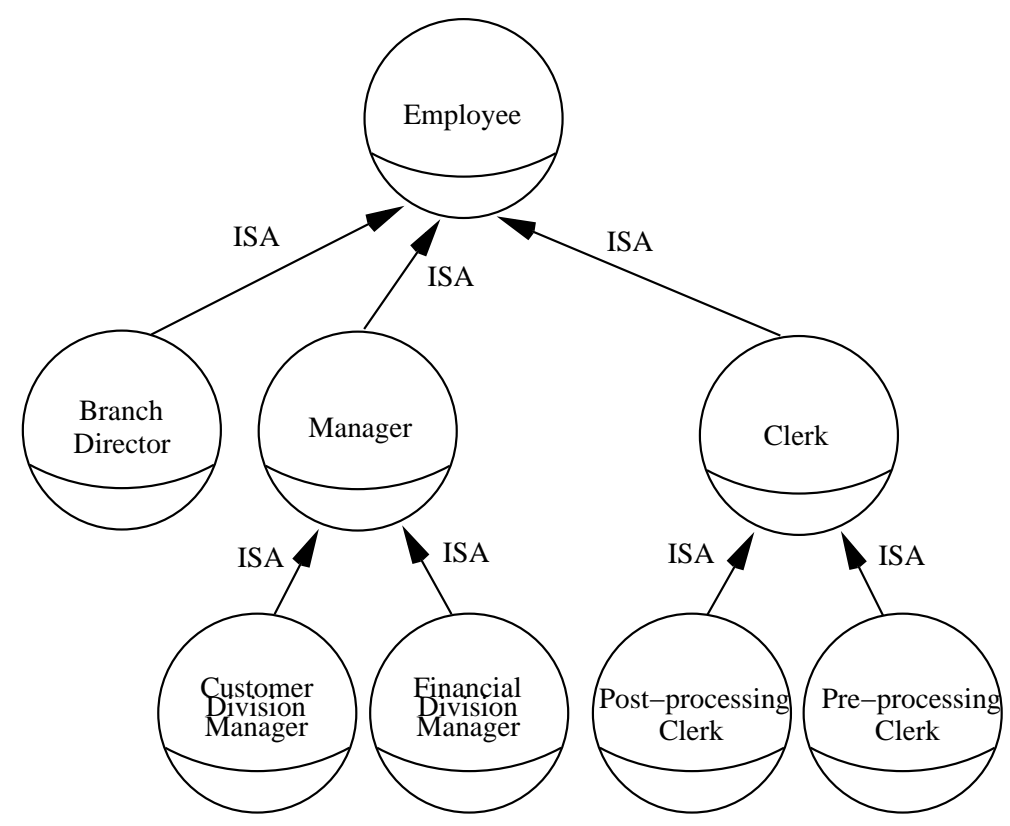

Figure 2 Role Specialization Hierarchy

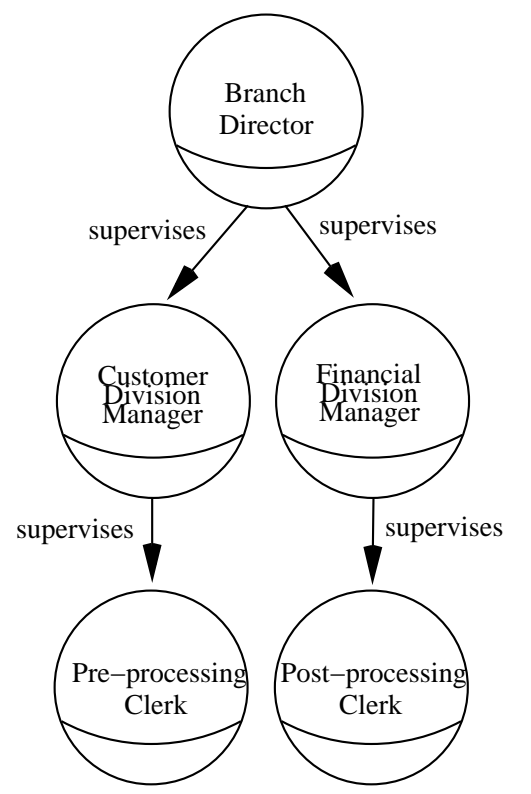

Figure 3 Role Supervision Hierarchy

\subsection{Goals, tasks and resources}

A goal represents a strategic interest of an actor. $\mathrm{Si}^{*}$, as well as $\mathrm{i}^{*}$, differentiates between hard (only goals hereafter) and soft goals. The latter have no clear definition or criteria for deciding whether they are satisfied or not, and are typically used to model non-functional requirements. According to [Chung et al., 2000], the different nature of fulfillment is underlined by saying that goals are satisfied, while softgoals are satisficed.

Goals can be fulfilled by means of tasks or resources. A task represents a particular course of actions that produces a desired effect. A task can be executed in order to satisfy a goal or satisfice a softgoal. A resource represents a physical or an informational entity without intentionality. A resource can be consumed or produced 
by a task. Figure 4 depicts the graphical representation of goals, softgoals, tasks, and resources.
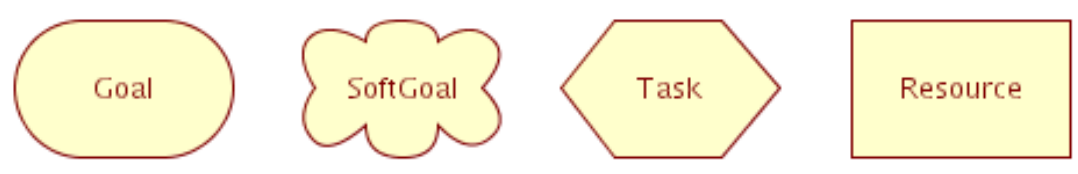

Figure $4 \mathrm{Si}^{*}$ graphical representation of goal, softgoal, task, and resource

$\mathrm{Si}^{*}$ is based on the idea of building a model of the system that is incrementally refined and extended. Specifically, goal modeling consists of refining goals and eliciting new social relationships among actors. Goals are analyzed from the perspective of single actors using three techniques, namely $A N D / O R$ decomposition, contribution analysis, and means-end analysis. In particular, AND/OR decomposition combines AND and OR refinements of a root goal into subgoals, modeling a finer goal structure. In essence, AND-decomposition is used to define the process for achieving a goal, while OR-decomposition defines alternatives for achieving a goal. Contribution analysis identifies goals and tasks that contribute positively or negatively in the fulfillment of the goal to be analyzed. Means-end analysis aims at identifying goals, softgoals, tasks, and resources that provide means for achieving a goal.

Example 2 One of the services offered by the bank is to offer loans. The provisioning of such a service contributes to increase bank profits. The bank AND-decomposes offer loans into identify customers, manage the loan process, sell the loan. These subgoals can be further decomposed until a plan to fulfill them is identified. For instance, getting customer data can be achieved by executing tasks insert customer identifier and retrieve customer data. Figure 5 shows the goal diagram derived applying goal analysis to offer loans. 


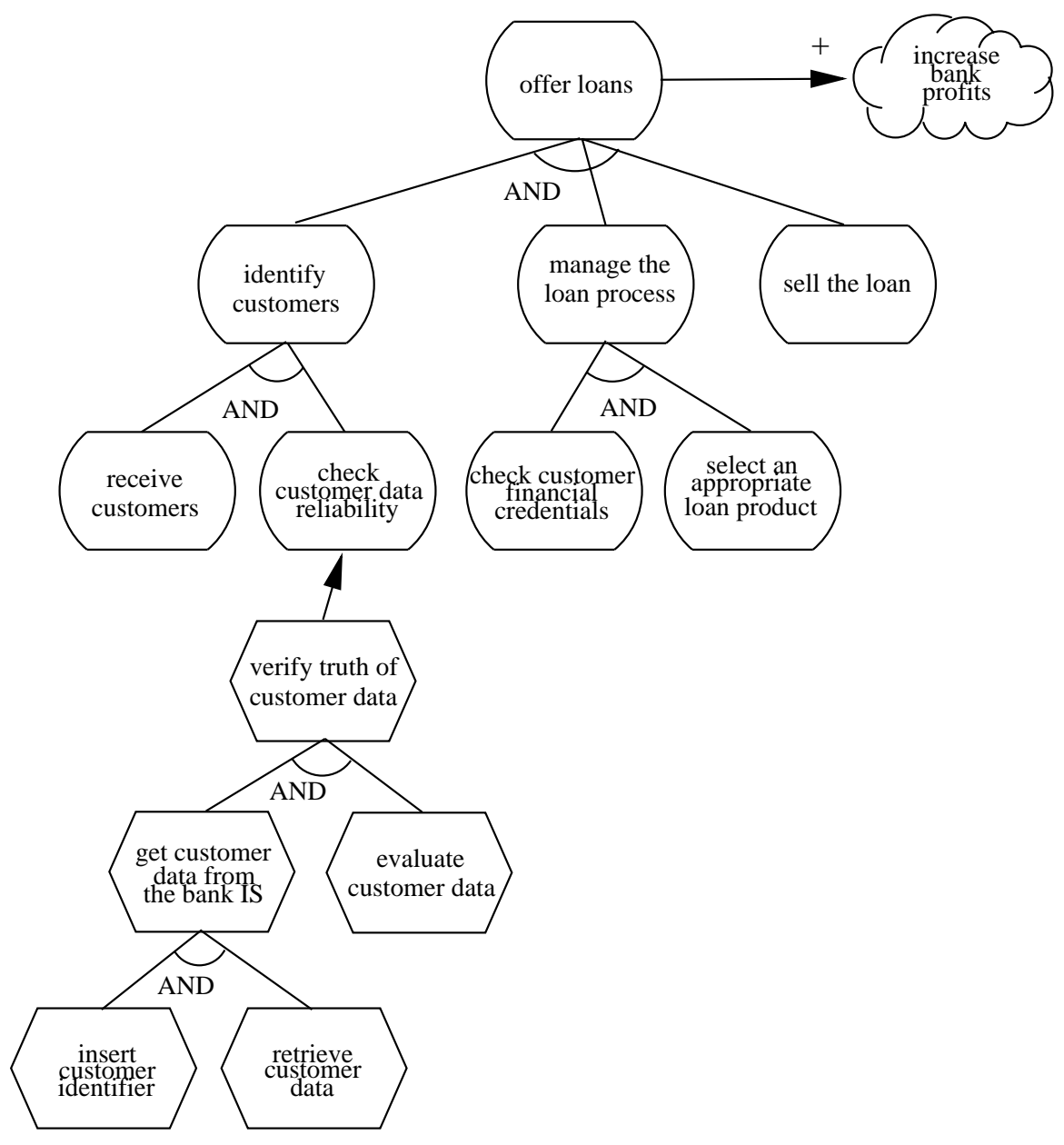

Figure 5 Goal diagram

\subsection{Objectives, Entitlements, and Capabilities}

The first intuition in modeling security aspects of information systems is to distinguish between actors who want access to a resource, fulfillment of a goal or execution of a task, from actors who have the capabilities to do any of the above, and - last but not least - actors who are entitled to do any of the above. Essentially, every actor is defined along with a set of objectives, capabilities, and entitlements.

Objectives, entitlements and capabilities of actors are modeled through relations between actors and services, namely require, be entitled, and provide.

Require indicates that an actor intends to achieve a goal, execute a task, or requires a resource.

Be entitled indicates that an actor is the legitimate "owner" of a goal, a task, or a resource. The basic idea is that an owner has full authority concerning access and disposition over his entitlements.

Provide indicates that the actor has the capability to achieve a goal, execute a task, or deliver a resource. 
The distinction between being entitled and providing allows us to model situations where the actor that has the capabilities to fulfill a goal is different from the one that has the permission to do it.

Example 3 According to data protection legislation, a customer is entitled to control the use of his personal data. The pre-processing clerk is appointed to identify customers. Thereby, he needs to access customer information to achieve his duties. However, he does not directly interact with the customer, but he retrieves such data from the bank IT system (that it has been designed to provide customer information to employees of the bank). Thus, the bank should seek the consent of the customer for granting access to the customer's data to all employees assigned to him.

In the graphical representation we represent relations require, be entitled, and provide as edges between an actor and a service, labeled by $\mathbf{R}, \mathbf{O}$ and $\mathbf{P}$, respectively.

\subsection{Trust and Delegation}

$\mathrm{Si}^{*}$ supports the notion of delegation in order to model the transfer of entitlements and responsibilities from an actor to another. Thus, delegation is a ternary relation among two actors (the delegator and the delegatee) and a goal, task or resource (the delegatum).

Example 4 A pre-processing clerk is interested in gathering customer data, for which he depends on the bank IT system. However, customer personal information is an entitlement of the customer himself. The customer delegates the permission to provide his data to the bank IT system on condition that they are not disclosed to third parties.

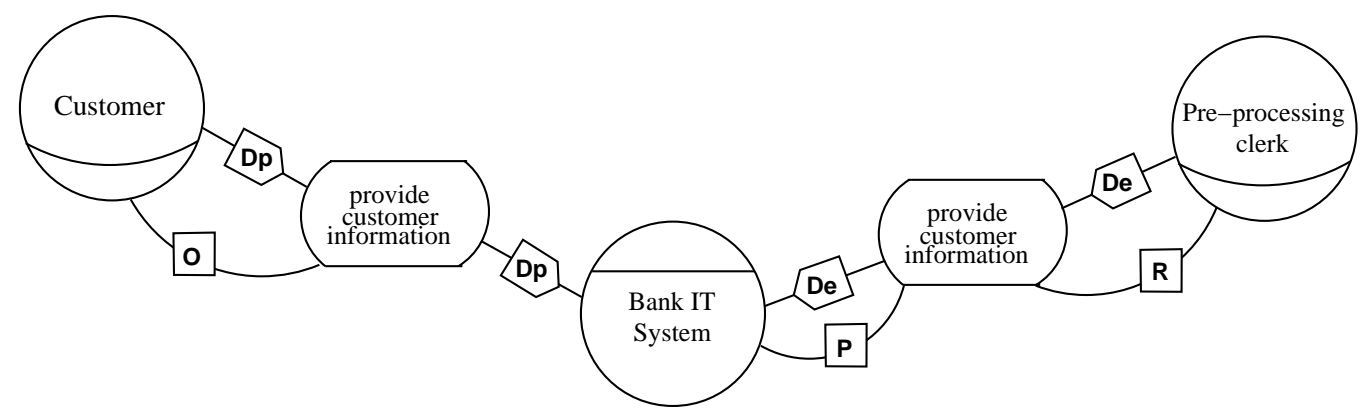

Figure 6 Delegation

In this scenario (Figure 6), there is a difference of relationship between the preprocessing clerk and the bank IT system and between the customer and the bank IT system. This difference is based on the type of delegation used in the two relationships. Thereby, we introduce a conceptual refinement of delegation, that will allow us to capture and model important security facets.

Delegation of execution indicates that one actor delegates to other actors the responsibility to achieve a goal, execute a task, or deliver a resource. This would be matched, for instance, by a call to an external procedure. In this setting, the delegatee will take care of the achievement of the goal, execution of the task, or delivery of the resource. At the same time, the delegator becomes vulnerable. 
Actually, the delegator has no warranty that the delegatee will achieve the goal, execute the task, or deliver the resource.

Delegation of permission indicates that one actor delegates to other actors the permission to achieve a goal, execute a task, or use a resource. This would be matched by issuing a delegation certificate, such as digital credential or a letter. In this setting, the delegatee is entitled to achieve the goal, execute the task, or use the resource. At the same time, the delegator becomes vulnerable. Actually, the delegator has no warranty that the delegatee will not misuse the delegatum.

In the graphical representation of Figure 6 we represent these relationships as edges respectively labeled $\mathbf{D e}$ and $\mathbf{D p}$.

Example 5 The customer delegates the permission to the bank IT system to provide only information relevant for the required service. On the other hand, the preprocessing clerk, who wants customer data, delegates the execution of his goal to the bank IT system. According to the pre-processing clerk, the bank IT system should provide the required information. He is not interested in what the bank IT system does with the customer consent, apart from getting his information. The clerk's major concern would be that tasks are delegated to people that can actually do them, whereas the customer would be concerned that his data are given to people who will not misuse the permissions they have acquired.

Further, we want to separate the concepts of trust and delegation, as we might need to model systems where some actors must delegate permission or execution to untrusted actors. Trust represents the willingness to accept vulnerability based on positive expectations about the behavior of another actor [Mayer et al,. 1995, Rousseau et al., 1998]. It is related to belief in honesty, trustfulness, competence, and reliability [Castelfranchi and Falcone, 1998, McKnight and Chervany, 1996] and it is to build collaboration between humans and organizations [Axelrod, 1984]. Trust is an important aspect for making decisions on security. This positive assumption about the intentions of another actor allows the system to economize on information processing and protection mechanisms. Trust supports system designers in making decisions more efficiently by simplifying the acquisition and interpretation of information. Trust also drives designers by suggesting protection mechanisms and procedures that are most practicable under the assumption that the trusted counterpart will not exploit vulnerabilities.

Similarly to delegation, we represent trust as a ternary relation among two actors (the trustor and the trustee) and a goal, a task or a resource. The object around which the trust relationship centers is called trustum. Also in this case it is convenient to have a suitable distinction for trust in managing permission and trust in managing execution.

Trust of execution indicates the belief of one actor on the capabilities of the other actor. In general, by trusting in execution another actor for a trustum, an actor is sure that the trustee will achieve the goal, perform the task, or furnish the resource.

Trust of permission indicates the belief of one actor that the other does not misuse a goal, a task, or some resource. By trusting in permission another actor for a trustum, an actor is sure that the trustum is properly used. 
In the graphical representation we represent these relationships as edges respectively labeled Te and Tp.

\section{A Formal Ontology}

To define a formal semantics for the new primitives, we use the Answer Set Programming (ASP) paradigm [Leone et al., 2006]. The ASP paradigm is based on the concepts of facts and rules expressed as Horn clauses and evaluated using the stable model semantics ${ }^{2}$. Facts are atomic statements representing the extensional description of the system. Rules can be axioms or properties: axioms are used to complete the extensional description of the system, whereas properties correspond to integrity constraints and are used to verify requirements consistency.

\subsection{Predicates}

Our setting distinguishes two types of predicates: intensional and extensional. Extensional predicates (Table 1) correspond to the edges and circles drawn by the requirements engineer during the modeling phase and are used to formalize the intuitive description of the system. Intensional predicates (Table 2) are determined with the help of rules by the reasoning system.

\subsubsection{Extensional Predicates}

For an automatic and precise analysis of requirements, graphical diagrams need to be translated in formal specifications. This has spurred us to define an extensional predicate for each primitive concept. Next, this set of predicates is presented and a summary is given in Table 1 .

\begin{tabular}{|l|}
\hline Type Predicates \\
\hline service(Service:s) \\
goal(Goal:g) \\
task(Task:t) \\
resource(Resource:r) \\
actor(Actor:x) \\
agent(Agent:a) \\
role(Role:p) \\
\hline Goal Analysis \\
\hline AND_decomp(Service:s,Service:s1,Service:s2) \\
OR_decomp(Service:s,Service:s1,Service:s2) \\
pos_contribution(Service:s1,Service:s2) \\
neg_contribution(Service:s1,Service:s2) \\
means_end(Service:s1,Service:s2) \\
\hline Association Relations \\
\hline play(Agent:a,Role:p) \\
is_a(Role:p,Role:q) \\
supervises(Role:p,Role:q) \\
is_part_of(Actor:a,Actor:b) \\
\hline
\end{tabular}

2 We assume that the reader is familiar with such concepts. Otherwise see [Leone et al., 2006] for a tutorial. 


\begin{tabular}{|l|}
\hline Actor Properties \\
\hline requires(Actor:a,Service:s) \\
owns(Actor:a,Service:s) \\
provides(Actor:a,Service:s) \\
\hline Delegation and Trust \\
\hline delegate(perm, $x, y, s)$ \\
delegate(exec, $x, y, s)$ \\
trust(perm,x,y,s) \\
trust(exec, $x, y, s)$ \\
\hline
\end{tabular}

Table 1 Extensional predicates

- Type Predicates: The unary predicates goal, task and resource are used respectively for identifying goals, tasks, and resources. Actually, some systems for ASP reasoning need them as explicit predicates; others allow their definition as types. For sake of compactness, we will use the unary predicate service when it is not necessary to distinguish among goals, tasks, and resources. We shall use letters $S, G, T$ and $R$ possibly with indexes as variables ranging over services, goals, tasks and resources, respectively. The unary predicates agent and role are used respectively for identifying agents, and roles. For sake of compactness, we introduce the unary predicate actor when is not necessary to distinguish among them. We shall use letters $X, Y$ and $Z$ as variable to indicate generic actor, $A, B$ and $C$ as variables to indicate agents, and $P, Q$ and $V$ as variables to indicate roles.

- Goal Analysis: Predicates AND_decomp and OR_decomp are used to model AND- and OR-decomposition, respectively. Predicates pos_contribution and neg_contribution are used to model positive and negative contribution, respectively. Finally, means_end states that a service provides means for achieving a goal with respect to the perspective of an actor.

- Association Relations: Predicate play identifies the role played by an agent. Predicate is_a is used to build specialization role hierarchies, whereas predicate supervises is used to build the organization chart of the system. Finally, predicate is_part_of identifies the sub-components of an actor.

- Actor Properties: Predicate require identifies the objectives of actors, provide the capabilities of actors, and entitled the legitimate owner of services.

- Delegation and Trust: Predicates delegate (perm,x,y,s) and delegate (exec,x,y,s) correspond to delegation of permission and delegation of execution, respectively. Predicates trust(perm,x,y,s) and trust(exec,x,y,s) correspond to trust of permission and trust of execution, respectively.

\subsubsection{Intensional Predicates}

The intuitive description of the system is not sufficient for an accurate verification of the system [Giorgini et al., 2006]. To derive the right conclusions from an intuitive model, such a model is completed using rules. To distinguish the relations drawn by the requirements engineer from the ones derived by the system, we introduce a set of intensional predicates. Next, we present such predicates and a summary is given in Table 2. 


\begin{tabular}{|c|}
\hline $\begin{array}{l}\text { AND_subservice(Service:s1,Service:s2) } \\
\text { OR_subservice(Service:s1,Service:s2) }\end{array}$ \\
\hline Actor Properties \\
\hline $\begin{array}{l}\text { aims(Actor:a,Service:s) } \\
\text { has perm (Actor:a,Service:s) }\end{array}$ \\
\hline Trust \\
\hline $\begin{array}{l}\text { trustChain(perm,Actor: } x, \text { Actor:y,Service:s) } \\
\text { trustChain(exec,Actor:x,Actor:y,Service:s) }\end{array}$ \\
\hline Confidence and Need-to-Know \\
\hline $\begin{array}{l}\text { in_charge(Actor:x,Service:s) } \\
\text { fulfills(Actor:x,Service:s) } \\
\text { can_satisfy(Actor:x,Service:s) } \\
\text { can_execute(Actor:x,Service:s) } \\
\text { confident(_atisfy,Actor:x,Service:s) } \\
\text { confident(execute,Actor:a,Service:s) } \\
\text { confident(owner,Actor:x,Service:s) } \\
\text { need_to_have_perm(Actor:x,Service:s) }\end{array}$ \\
\hline
\end{tabular}

\section{Table 2: Intensional Predicates}

- Goal Analysis: These predicates identify the relations among services in terms of subparts. Predicates subservice, OR subservice and AND subservice respectively identifies a subservice, OR-subservice and AND-subservice of a service. More specific predicates should be introduced for goal, task and resource decomposition.

- Actor Properties: Predicate aims identifies direct and indirect objectives of actors and has_perm identifies direct and indirect entitlements of actors.

- Trust: Trust relations can be combined to build trust chains. In particular, trustChain(perm,x,y,s) and trustChain(perm, $x, y, s)$ chains of trust of permission and trust of execution, respectively.

- In charge and fulfill: Predicate in charge identifies actors who take care of the final delivery of a service and fulfills identifies actors who are actually willing to deliver a service.

- Confidence of execution: This set of predicates is used to capture the notions of confidence from the point of view of the requester. Predicate can_satisfy identifies actors who delegate their objectives to actors who have the capabilities to fulfill them. Predicate can_execute identifies actors who delegate their objectives to actors who will fulfill them. confident(satisfy, $x, s$ ) identifies actors that are confident that a service can be satisfied. confident(execute, $x, s$ ) identifies actors that are confident that a service will be fulfilled. This is the case if an actor knows that all delegations have been done to trusted actors and that the actor, who will ultimately deliver the service, has permission to do so.

- Confidence of entitlements: From the point of view of the owner, confidence means that the owner is confident that the permission that he has delegated will not be misused. Thereby, confident(owner,x,s) holds if owner $x$ is confident to give the permission on service $s$ is granted only to trusted actors.

- Need-to-Know: Current privacy and data protection legislation requires that information is unavailable to actors except those who need legitimately to know (need-to-know principle). Essentially, this corresponds to the desire of owners to delegate permissions to providers only if the latter actually do need the permission. Predicate need_to_have_perm is used to capture this idea. 


\subsection{Axioms}

This section describes the axioms that define the semantics underlying $\mathrm{Si}^{*}$. They are used to complete the extensional description of the system. ${ }^{3}$

\subsubsection{Trust}

Table 3 presents the set of axioms for propagating trust relations along chains and service refinement. ${ }^{4}$

\begin{tabular}{|c|c|}
\hline \\
\hline \multicolumn{2}{|c|}{\begin{tabular}{|l}
\multicolumn{1}{r}{ Trust } \\
$\mathrm{T} 1$
\end{tabular}} \\
\hline $\mathrm{T} 2$ & trustChain $($ exec, $X, Y, S) \leftarrow \operatorname{trust}($ exec $, X, Z, S) \quad$ trustChain(exec, $X, Y, S)$ \\
\hline $\mathrm{T} 3$ & trustChain $($ exec, $X, Y, S 1) \leftarrow$ subservice $(S, S 1)$ trustChain $($ exec, $X, Y, S)$ \\
\hline $\mathrm{T} 4$ & trustChain $($ perm $, X, Y, S) \leftarrow$ trust $($ perm $, X, Y, S)$ \\
\hline $\mathrm{T} 5$ & trustChain $($ perm, $X, Y, S) \leftarrow \operatorname{trust}($ perm $, X, Z, S) \quad$ trustChain $($ perm $, X, Y, S)$ \\
\hline T6 & trustChain $($ perm $, X, Y, S) \leftarrow$ subservice $(S, S 1) \quad$ trustChain(perm, $X, Y, S 1)$ \\
\hline
\end{tabular}

Table 3: Trust Propagation

- Trust (T1-6) These axioms are used to builds trust chains. T1-2 are used to build trust chains for execution. T3 propagates trust relationships from a service to its parts. T4-5 are used to build trust chains for permission. T6 propagate along service refinements. If an actor trusts that another will not overstep the set of actions required to fulfill a part of a service, then the first can trust the last will not overstep the set of actions required to fulfill the service. Thereby, trust of permission flows bottom-up with respect to goal refinements.

\subsubsection{Fulfillment, Confidence, and Need-to-Know}

Tables 4 and 5 present the set of axioms for identifying entitlements and responsibilities of actors; also, actors who will fulfill services and actors who are confident that their objectives will be fulfilled and their entitlements will not misused.

\begin{tabular}{|l|}
\hline Aims \\
\hline AP1 $\quad \operatorname{aims}(X, S) \leftarrow$ requires $(X, S)$ \\
AP2 $\quad \operatorname{aims}(X, S) \leftarrow$ delegate $(\operatorname{exec}, Y, X, S) \quad \operatorname{aims}(Y, S)$ \\
AP3 $\quad \operatorname{aims}(X, S) \leftarrow$ subservice $(S 1, S) \quad \operatorname{aims}(Y, S)$ \\
\hline Has permission \\
\hline AP4 has_perm $(X, S) \leftarrow \operatorname{requires}(X, S)$ \\
\hline
\end{tabular}

3 We do not present here the axiomatization for the user-role assignment and goal analysis. We refer to [Giorgini et al., 2005] for it.

$4 \quad$ For the sake of simplicity we do not deal with the question of depth here. See $\mathrm{Li}$ et al. [Li et al, 2003] for an account of trust with depth. What has emerged from several case studies is that depth is less important than qualifications such as "only to members of the same office”. 


\begin{tabular}{|c|c|}
\hline AP5 & $\begin{array}{l}\text { has_perm }(X, S) \leftarrow \text { delegate }(\text { perm, } Y, X, S) \quad \text { has_perm }(Y, S) \\
\text { has_perm }(X, S) \leftarrow \text { subservice }(S 1, S) \quad \text { has_perm }(Y, S)\end{array}$ \\
\hline \multicolumn{2}{|c|}{ In charge } \\
\hline AP7 & in_charge $(X, S) \leftarrow \operatorname{aims}(X, S)$ provides $(X, S)$ \\
\hline \multicolumn{2}{|l|}{ Fulfill } \\
\hline AP8 & fulfills $(X, S) \leftarrow$ in_charge $(X, S)$ has_perm $(X, S)$ \\
\hline \multicolumn{2}{|c|}{ Can satisfy } \\
\hline AP9 & can_satisfy $(X, S) \leftarrow$ in_charge $(X, S)$ \\
\hline AP10 & can_satisfy $(X, S) \leftarrow$ delegate $($ exec $, X, Y, S) \quad$ can_satisfy $(Y, S)$ \\
\hline AP11 & can_satisfy $(X, S) \leftarrow O R \_$subservice $(S 1, S) \quad$ can_satisfy $(X, S 1)$ \\
\hline AP12 & can_satisfy $(X, S) \leftarrow A N D \_d e c o m p(S, S 1, S 2) \quad$ can_satisfy $(X, S 1) \quad$ can_satisfy $(X, S 2)$ \\
\hline \multicolumn{2}{|c|}{ Can execute } \\
\hline AP13 & can_execute $(X, S) \leftarrow$ fulfills $(X, S)$ \\
\hline AP14 & can_execute $(X, S) \leftarrow$ delegate $($ exec, $X, Y, S) \quad$ can_execute $(Y, S)$ \\
\hline AP15 & can_execute $(X, S) \leftarrow O R \_$subservice $(S 1, S) \quad$ can_execute $(X, S 1)$ \\
\hline AP16 & 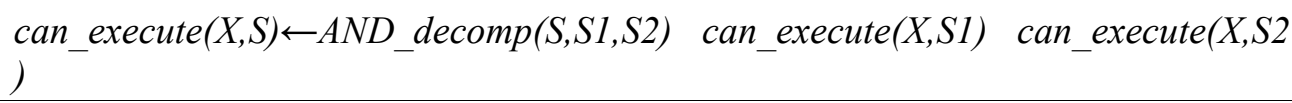 \\
\hline
\end{tabular}

\section{Table 4: Entitlements and Objectives Transfer and Fulfillment}

- Aims (AP1-3) AP1 states that if an actor requests a service fulfilled, he aims its fulfillment. AP2 states that if an actor requires a service delivered and delegates its execution to another actor, the delegatee takes care of its fulfillment so that it becomes his objective. Finally, AP3 propagates objectives through service refinement.

- Has permission (AP4-6) The owner of a service has full authority concerning access and disposition of it. Thus, AP4 states that if an actor owns a service, he is entitled to deliver it. AP5 states that if an actor is entitled to deliver a service and delegates the permission to another actor, the delegatee is entitled to deliver the service. Finally, AP6 propagates entitlements through service refinement.

- In charge (AP7) An actor will take charge of the fulfillment of a service if he has the capabilities to fulfill it and it belongs to his objectives.

- Fulfill (AP8) An actor will fulfill a service if he has taken charge of its fulfillment and has the permission to fulfill it.

- Can satisfy (AP9-12) An actor can satisfy his objectives if either he has taken charge of them (AP9) or has delegated it to someone who will take charge of them (AP10). Service decompositions are accounted for through axioms AP11-12. If an actor can satisfy at least one of the OR-subservices of a service, then he can satisfy the root service. Dual axiom holds for ANDdecompositions.

- Can execute (AP13-16) These axioms is used to identify actors that actually can deliver a service by combining execution with permission. An actor can fulfill his objectives if either he will fulfill them directly (AP13) or has delegated its execution to someone who will fulfill them (AP14). Service decompositions are accounted for through axioms AP15-16. If an actor can execute at least one of the OR-subservices of a service, then he can execute the root service. Dual axiom holds for AND-decompositions. 


\begin{tabular}{|c|c|}
\hline \multicolumn{2}{|c|}{ Confident of satisfaction } \\
\hline AP17 & confident $($ satisfy, $X, S) \leftarrow$ in_charge $(X, S)$ \\
\hline AP18 & $\begin{aligned}\text { confident(satisfy, } X, S) \leftarrow & \text { delegate }(\text { exec, } X, Y, S) \text { trustChain(exec, } X, Y, S) \\
& \text { confident }(\text { satisfy, } X, S)\end{aligned}$ \\
\hline AP19 & confident(satisfy, $X, S) \leftarrow O R \_$subservice $(S 1, S) \quad$ confident(satisfy, $\left.X, S 1\right)$ \\
\hline AP20 & $\begin{aligned}\text { confident(satisfy, } X, S) \leftarrow & A N D \_\operatorname{decomp}(S, S 1, S 2) \quad \text { confident(satisfy,X,S1) } \\
& \text { confident(satisfy,X,S1) }\end{aligned}$ \\
\hline \multicolumn{2}{|c|}{ Confident of execution } \\
\hline AP21 & confident (execute, $X, S) \leftarrow$ fulfills $(X, S)$ \\
\hline AP22 & 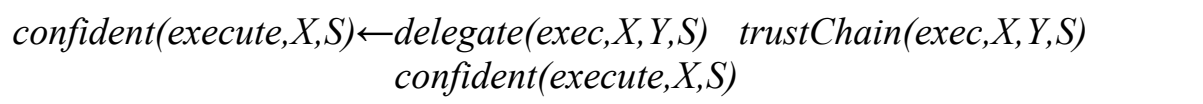 \\
\hline AP23 & confident $($ execute $, X, S) \leftarrow O R \_$subservice $(S 1, S) \quad$ confident(execute, $\left.X, S 1\right)$ \\
\hline AP24 & $\begin{aligned} \text { confident }(\text { execute, } X, S) \leftarrow & A N D \_ \text {decomp }(S, S 1, S 2) \text { confident }(\text { execute, } X, S 1) \\
& \text { confident }(\text { execute }, X, S 1)\end{aligned}$ \\
\hline \multicolumn{2}{|c|}{ Confident of entitlements } \\
\hline AP24 & confident $($ owner $, X, S) \leftarrow o w n s(X, S) \quad$ not diffident $(X, S)$ \\
\hline AP26 & diffident $(X, S) \leftarrow$ delegate $($ exec, $X, Y, S)$ not trustChain(perm, $X, Y, S)$ \\
\hline AP27 & $\operatorname{diffident}(X, S) \leftarrow \operatorname{delegate}($ exec $, X, Y, S) \quad \operatorname{diffident}(X, S)$ \\
\hline AP28 & $\operatorname{diffident}(X, S) \leftarrow$ subservice $(S 1, S) \quad \operatorname{diffident}(X, S)$ \\
\hline \multicolumn{2}{|c|}{ Need to know } \\
\hline AP29 & need_to_have_perm $(X, S) \leftarrow$ in_charge $(X, S)$ \\
\hline AP30 & $\begin{aligned}\text { need_to_have_perm }(X, S) \leftarrow \text { delegate(perm, } X, Y, S) \text { not other_delegater }(X, Y, S) \\
\text { need_to_have_perm }(Y, S)\end{aligned}$ \\
\hline AP31 & $\begin{aligned} \text { other_delegater }(X, Y, S) \leftarrow \text { delegate }(\text { perm }, X, Y, S) \quad \text { delegate }(\text { perm }, Z, Y, S) \\
\\
\text { need_to_have_perm }(Z, S) \quad X \neq Z\end{aligned}$ \\
\hline
\end{tabular}

\section{Table 5: Confidence and Need-to-Know}

- Confidence of satisfaction (AP17-20) An actor is confident that its objectives will be satisfied if he takes care of them (AP17) or he has delegated their execution to a trusted actors who take care of them (AP18). Axioms AP19-20 specify how confidence of satisfy is propagated upwards along service decomposition.

- Confidence of execution (AP21-24) An actor is confident to fulfill his objectives if he fulfills them by himself (AP21) or he has delegated their execution to trusted actors who will fulfill them (AP22). Axioms AP23-24 propagate confidence of execution upwards along service decomposition.

- Confidence of entitlements (AP25-28) An owner is confident, if there is no likely misuse of his permission. It can be seen that there is an intrinsic double negation in the statement. We model it using a predicate diffident. At any point of delegation of permission, the delegating agent is diffident, if the delegation is being done to an untrusted agent (AP26) or if the delegatee could be diffident himself (AP27). AP28 propagates diffidence upwards along service decomposition.

- Need to Know (AP29-31) These axioms defines the semantics of intensional predicates that are necessary to analyze need-to-know properties. This set of axioms also captures the possibility of having alternate paths of permission delegations through predicate other_delegater. In this case the formal analysis will not yield one model but multiple models in which only one path of 
delegation is labeled by the need-to-have property and the others are not. Essentially, AP30 introduces non-determinism, so it makes search and verification harder.

\section{Analysis and Verification}

The suggested primitives were sufficient to deal with most of the security organizational requirements we encountered. For instance, it has been shown that $\mathrm{Si}^{*}$ is able to cope with the complexity of a real ISO-17799-like case study [Massacci et al., 2005]. Security requirements are verified using properties. Such properties are defined in form of patterns that have to be checked. In ASP, they are represented as integrity constraints that a good design should satisfy. If the set of features is not consistent, i.e., properties cannot all be simultaneously satisfied, the system is not secure. In this setting, ASP solvers will return a model only if the design complies with all properties. Indeed, they do not return inconsistent model. Table 6 presents the basic set of properties.

\begin{tabular}{|c|c|}
\hline \multicolumn{2}{|c|}{ Authorization } \\
\hline Pro1 & $\leftarrow$ delegate $($ perm, $X, Y, S) \quad$ not trustChain $($ perm, $X, Y, S)$ \\
\hline Pro2 & $\leftarrow$ delegate $($ perm, $X, Y, S) \quad$ not has_perm $(X, S)$ \\
\hline Pro3 & $\leftarrow$ owns $(X, S) \quad$ not confident (owner, $X, S)$ \\
\hline \multicolumn{2}{|c|}{ Availability } \\
\hline Pro4 & $\leftarrow$ delegate $($ exec $, X, Y, S) \quad$ not trustChain $($ exec, $X, Y, S)$ \\
\hline Pro5 & $\leftarrow$ requests $(X, S) \quad$ not can_satisfy $(X, S)$ \\
\hline Pro6 & $\leftarrow$ requests $(X, S) \quad$ not can_execute $(X, S)$ \\
\hline Pro7 & $\leftarrow$ requests $(X, S)$ not confident(satisfy, $X, S)$ \\
\hline Pro8 & $\leftarrow$ requests $(X, S)$ not confident(execute, $X, S)$ \\
\hline Pro9 & $\leftarrow$ need_to_have_perm $(X, S)$ not has_perm $(X, S)$ \\
\hline \multicolumn{2}{|c|}{ Privacy } \\
\hline Pro10 & $\leftarrow$ has_perm $(X, S)$ not need_to_have_perm $(X, S)$ \\
\hline
\end{tabular}

Table 6: Security Properties

- Authorization (Pro1-3) Pro1 is used to verify if an actor has delegated permission to another actor that he trusts. This corresponds to the assumption that trust is a prerequisite for delegation. Pro2 verifies whether an actor who delegates the permission to deliver a service is entitled to do it. Pro3 verifies that the owner of the service has to be confident to give the service only to trusted actors.

- Availability (Pro4-9) Pro4 is used to verify if an actor has delegated the execution of a service to another actor that he believes has the capability to achieve it. Other properties are used to verify the expectancies of actors. Pro56 check if actors can satisfy and execute the required services. Pro7-8 deal with the notion of confidence and, in particular, verify whether a requester is confident to satisfy and execute required services, respectively. Pro9 verifies that actors who need the permission for performing their duties have such permission.

- Privacy (Pro10) Pro10 verifies that actors, who have the permission on a service, actually need such permission. 


\section{Conclusion}

This chapter has proposed an ontology intended to model security at an organizational level. Our proposal is founded on the concepts of ownership, trust and delegation. Moreover, by making explicit the objectives, entitlements and capabilities of stakeholder and system actors, as well as their delegations to other actors, we can analyze functional and security requirements at the same time. The proposed concepts proved up to the challenge, and revealed a number of pitfalls, especially when formal analysis techniques were applied [Massacci and Zannone, 2006].

We are currently extending the ontology to capture behavioral aspects of the system. Such an extension has two implications. On one hand, it allows system designers to capture more sophisticated security properties. On the other hand, such concepts support the (semi-)automatic derivation of business processes from the requirements model.

Another direction under investigation involves the enrichment of the $\mathrm{Si}^{*}$ ontology with concepts necessary for capturing privacy concerns. According to existing privacy legislations in many countries (e.g., the US Privacy Act and the EU Privacy Directive), privacy is mainly maintained by controlling the usage of information. This requires that information be linked to the functional requirements of the original application. Following this trend, researchers have recently proposed frameworks for specifying and enforcing privacy policies [OASIS, 2005, Ashley et al., 2003, Cranor et al., 2002]. These proposals introduce concepts appropriate for modeling privacy policies such as the ones of purpose and obligation. However, they do not support policy writers in the analysis of organizational requirements and leave them to manually define privacy policies. Our objective is to bridge the gap between the requirements analysis and policy specification by deriving privacy policies directly from the requirements model.

\section{Acknowledgments}

We thank Nicola Guarino and ISTC-CNR Laboratory for Applied Ontology in Trento for many useful discussions. This work was partly supported by the projects FIRBTOCAI, IST-FP6-FET-IP-SENSORIA, IST-FP6-IP-SERENITY, and PATMOSTRO.

\section{References}

AMICE Consortium (1993). Open System Architecture for CIM. Springer-Verlag.

Anderson, R. (1994). Why cryptosystems fail. Communications of the ACM, 37(11):32-40.

Ashley, P., Hada, S., Karjoth, G., Powers, C., and Schunter, M. (2003). Enterprise Privacy Authorization Language (EPAL 1.2). W3C Recommendation. Available at http://www.w3.org/Submission/EPAL/.

Axelrod, R. (1984). The Evolution of Cooperation. Basic Books.

Basin, D., Doser, J., and Lodderstedt, T. (2006). Model Driven Security: from UML Models to Access Control Infrastructures. TOSEM, 15(1):39-91. 
Bernus, P. and Nemes, L. (1996). A Framework to Define a Generic Enterprise Reference Architecture and Methodology. Computer Integrated Manufacturing Systems, 9(3):179-191.

Bresciani, P., Giorgini, P., Giunchiglia, F., Mylopoulos, J., and Perini, A. (2004). TROPOS: An Agent-Oriented Software Development Methodology. JAAMAS, 8(3):203-236.

Bryce, M. and Associates (2006). PRIDE-EEM Enterprise Engineering Methodology. http://www.phmainstreet.com/mba/pride/eemeth.htm.

Castelfranchi, C. and Falcone, R. (1998). Principles of trust for MAS: Cognitive anatomy, social importance and quantification. In Proceedings of $3 \mathrm{rd}$ International Conference on Multi-Agent Systems, pages 72-79. IEEE Computer Society Press.

Chung, L. K., Nixon, B. A., Yu, E., and Mylopoulos, J. (2000). Non-Functional Requirements in Software Engineering. Kluwer Publishing.

Cranor, L., Langheinrich, M., Marchiori, M., and Reagle, J. (2002). The Platform for Privacy Preferences 1.0 (P3P1.0) Specification. W3C Recommendation.

Dardenne, A., van Lamsweerde, A., and Fickas, S. (1993). Goal-directed Requirements Acquisition. Science of Computer Programming, 20:3-50.

Dignum, V. (2004). A model for organizational interaction: based on agents, founded in logic. $\mathrm{PhD}$ thesis, Universiteit Utrecht.

Emery, F. E. and Trist E. L. (1960). Socio-technical systems. In Management Sciences: Models and Techniques, volume 2, pages 83-97. Pergamon Press

Fuxman, A., Giorgini, P., Kolp, M., and Mylopoulos, J. (2001). Information systems as social structures. In Proceedings of the 2nd International Conference on Formal Ontology in Information Systems, pages 10-21. ACM Press.

Giorgini, P., Massacci, F., Mylopoulos, J., and Zannone, N. (2006). Requirements Engineering for Trust Management: Model, Methodology, and Reasoning. IJIS, 5(4):257-274.

Giorgini, P., Massacci, F., and Zannone, N. (2005). Security and Trust Requirements Engineering. In Foundations of Security Analysis and Design III - Tutorial Lectures, LNCS 3655, pages 237-272. Springer.

House of Lords (1999). Prince Jefri Bolkiah vs KPMG. 1 All ER 517. Available on www.parliament.the-stationeryoffice.co.uk.

Hübner, J. F., Sichman, J. S., and Boissier, O. (2002). A Model for the Structural, Functional, and Deontic Specification of Organizations in Multiagent Systems. In Proceedings of the 16th Brazilian Symposium on Artificial Intelligence, pages 118-128. Springer.

Jürjens, J. (2004). Secure Systems Development with UML. Springer-Verlag.

Lampson, B. W. (2004). Computer Security in the Real World. Computer, 37(6):3746. 
Leone, N., Pfeifer, G., Faber, W., Eiter, T., Gottlob, G., Perri, S., and Scarcello, F. (2006). The DLV System for Knowledge Representation and Reasoning. TOCL, 7(3):499-562.

Li, N., Grosof, B. N., and Feigenbaum, J. (2003). Delegation logic: A logic-based approach to distributed authorization. TISSEC, 6(1):128-171.

Liu, L., Yu, E. S. K., and Mylopoulos, J. (2003). Security and Privacy Requirements Analysis within a Social Setting. In Proceedings of the 11th IEEE International Requirements Engineering Conference, pages 151-161. IEEE Computer Society Press.

Masolo, C., Vieu, L., Bottazzi, E., Catenacci, C., Ferrario, R., Gangemi, A., , and Guarino, N. (2004). Social roles and their descriptions. In Proceedings of the 9th International Conference on the Principles of Knowledge Representation and Reasoning, pages 267-277. AAAI Press.

Massacci, F., Prest, M., and Zannone, N. (2005). Using a Security Requirements Engineering Methodology in Practice: The compliance with the Italian Data Protection Legislation. Computer Standards \& Interfaces, 27(5):445-455.

Massacci, F. and Zannone, N. (2006). Detecting Conflicts between Functional and Security Requirements with Secure Tropos: John Rusnak and the Allied Irish Bank. Technical Report DIT-06-002, University of Trento.

Mayer, R. C., J. H. Davis, F. D. Schoorman. 1995. An integrative model of organizational trust. Acad. Management Rev, 20(3):709-734.

McDermott, J. and Fox, C. (1999). Using Abuse Case Models for Security Requirements Analysis. In Proceedings of $A C S A C$ '99, pages 55-66. IEEE Press.

McKnight, D. H. and Chervany, N. L. (1996). The meanings of trust. Technical Report 96-04, MIS Research Center.

Michaely, R. and Womack, K. L. (1999). Conflict of interest and the credibility of underwriter analyst recommendations. Review of Financial Studies, 12(4):653686.

Moffett, J. D. (1998). Control principles and role hierarchies. In Proceedings of the 3rd ACM Workshop on Role-Based Access Control, pages 63-69. ACM Press.

OASIS (2005). eXtensible Access Control Markup Language (XACML) Version 2.0. OASIS Standard. Available at http://docs.oasisopen.org/xacml/2.0/access_control-xacml-2.0-core-spec-os.pdf.

Ponemon L. (2003). What Keeps Security Professionals Up At Night? Available at http://www.darwinmag.com/read/040103/threats.html.

Rousseau, D. M., S. B. Sitkin, R. S. Burt, C. Camerer. 1998. Not so different after all: A cross-discipline view of trust. Acad. Management Rev. 23(3) 393-404.

Sandhu, R. S., Coyne, E. J., Feinstein, H. L., and Youman, C. E. (1996). Role-based access control models. IEEE Computer, 29(2):38-47.

Sindre, G. and Opdahl, A. L. (2005). Eliciting security requirements with misuse cases. REJ, 10(1):34-44. 
Sommerville, I. (2001). Software Engineering. Addison-Wesley.

Stader, J. (1996). Results of the Enterprise Project. Technical Report AIAI-TR-209, University of Edinburgh.

Strens, R. and Dobson, J. (1993). How responsibility modelling leads to security requirements. In Proceedings of the 1993 New Security Paradigms Workshop, pages 143-149. ACM Press.

Tryfonas, T., Kiountouzis, E., and Poulymenakou, A. (2001). Embedding security practices in contemporary information systems development approaches. Information Management and Computer Security, 9:183-197.

Uschold, M., King, M., Moralee, S., and Zorgios, Y. (1998). The Enterprise Ontology. The Knowledge Engineering Review, 13(1):31-89.

$\mathrm{Yu}, \mathrm{E}$. (1996). Modelling strategic relationships for process reengineering. $\mathrm{PhD}$ thesis, University of Toronto. 